had been sent to a hospital without previous vaginal examination and operated upon in time.

To my understanding the efforts of Dr. Hirsch are creditable in so far that the early removal of complicated cases to hospitals and the omitting of unnecessary vaginal examinations would certainly bring about a decreasing puerperal morbidity and mortality. But to me it seems exaggerated to go so far as Hirsch wants to do. The new orientation that he so strongly advocates and wishes to introduce is already partly realized, and the surgical tenclencies in obstetrics are nothing new. I myself have performed Caesarean section in 20 out of 200 patients with placenta praevia. I believe that no experienced obstetrician would hesitate about Caesarean section if he was really convinced that this operation would confer a better prognosis without increasing the risks for mother or child. Neither do I believe in the necessity or possibility of transferring all cases to hospitals, as Hirsch insists upon. The majority of labours go well by means of natural forces alone, and there is indeed no branch of medicine that in ordinary circumstances can be performed so efficiently and safely in the patient's home as obstetrics.

Furthermore, Dr. Hirsch's handling of the statistical figures does not quite appeal to me. Generally speaking, it is difficult to get reliable statistics on this special point. 1 particularly wish to draw attention to the very thorough and excellent investigation of the Caesarean sections performed in Germany in 1928 which has been recently published by Winter. It really does not point in the same direction as Hirsch, but quite the contrary. For the present $I$ am thercfore of the opinion that Hirsch overrates the possibilities of Caesarean section, while he at the same time underrates what can be done by simpler methods without surgical intervention.

Another example of misjudging the Caesarean section has been given recently in Sweden, when a colleague put the question: "Why does not one perform that operation more frequently in order to shorten the labour and alleviate the pains of the mother?" I need not here go into a detailed criticism of such a proposal, which seems to indicate that the desire for more speed, which is such a characteristic feature of our days, threatens to extend into the domain of obstetrics.

I should venture to go farther and say that these and other efforts to extend surgical intervention in obstetries are indicative of a too superficial understanding of childbirth. Labour is decidedly a physiological process, the most difficult and strenuous that the human organism is able to perform. Who knows the deeper effects of such an enormous effort on the body of the mother herself? The coming into life of new individuals is associated not only with risks, but also with means of safety which may be considered as wellnigh perfect. When trying to follow and understand them we are reminded of the words of our great Limnaeus: "Deun omnipotentem a tergo transeuntem vidi." And we ought to remember, too, that nothing great and dear in life is attained without strugglo and suffering. If we try to reach the goal by easy byways, we may perhaps find that we have lost more than we have gained.

No; surgical intervention is not intended to shorten a physiological process, nor merely to alleviate it. It is permissible only after thorough consideration of the pros and cons. It requires of the obstetrician a patient observation and infallible recognition of commencing complications, the power of choosing the way of the greatest safety, and, not least, a sufficient surgical training. These qualities can be demonstrated by choosing a surgical procedure which is considered advisable or necessary, but they can also be just as well shown by abstaining from interfering in a physiological process if the interference is unnecessary
or risky.

\section{THE TREATMENT OF HYPERPIESIA.* BY}

\section{A. H. DOUTHWAITE, M.D., F.R.C.P.,} assistant PHYSICIAN, GUY'S hospital.

BFFore dealing with the details of treatment of this pievalent disease I propose bringing to your notice a medley of symptoms, any one or more of which may be indicative of its existence. In the first place, we find that cases of hyperpiesia may be divided into three types:

1. Menopausal, in which the common vasomoto: disturbances are only too apt to divert attention from the possibility of associated hyperpiesis. The subjects present increasing ohesity and other signs suggestive of hypothyroidism-for example, loss of hair, waxy colour of the skin, and subnormal temperature.

2. Plethoric, preponderating in males of 40 years or more, who are fat with florid facies and short, thick neck. Often this type has for years taken freely of food and alcohol, but sparingly of exercise; this, however, is by no means always the case. The majority suffer but little from symptoms of the disease, and form the greater part of those dying suddenly and unexpectedly.

3. Sallow, found chiefly in females, the disease com mencing generally at an earlier age than that of the first two types. The picture presented is that of the patient with visceroptosis and intestinal toxaemia. The skin is muddy in appearance, dark rings are present beneath the eyes, the tongue is thickly furred, and constipation is the rule. 'These people are thin, "wiry," energetic workers, seldom given to alimentary indiscretions, but over-conscientious in the performance of their tasks. This is the type which actually suffers so greatly from the disease which afflicts it, and which responds the least readily to treatment. I lay stress on its description, because the lean patient is prone to pass through our hands incorrectly diagnosed, the possibility of a raised liood pressure never having been considered.

* An address delivered before the Dover Division of the British Medical Association, May 11th, 1929.
We must recognize, also, that hyperpiesia is by no means a disease whose onset is peculiar to middle life. Genuine cases are met with in young adults and in adolescence-a fact which those of you whose work includes the routine examination of school children will confirm.

Lastly, in this connexion, should be mentioned the type of patient whose blood pressure rearing may lead to the diagnosis of hyperpiesia, yct in whom the hyperpiesis is transitory and the result of apprehension only. It is a matter of common knowlecige that the first estimation of blood pressure in a strange patient is unreliable, but in some cases nervousness may produce truly surprising results. Thus a male patient whom I saw recently had blood pressures of $220 / 130$. Suspecting this as a case of "fictitious" lyperpiesia, I took a series of readings during the next half-hour. They were 180/125, 160/120, 145/100. Actually he was suffering from peripheral neuritis, but he thought that the pains in his arm were due to angina pectoris, a disease of which a near relative had died. Whether this fcrm represents a potential hyperpiesia, a hyperpietic in embryo, is, I think, a point worthy of consideration.

\section{Symptoms.}

The variety of symptoms of hyperpiesia is one of the characteristics of the disease. It is therefore of importance that they should be briefiy considered, because many of them do not immediately suggest to the physician the presence of cardio-vascular disorder. I have arranged these roughly in order of descending frequency as noted ia one hundred consecutiro cases.

Shortness of breath on exertion and headache vie for pride of place. I have previously shown ${ }^{2}$ that the headache is of two types: one is the true dull ache, not uncommonly present even with the decline of high pressure; the other is an acute lancinating pain, the head feeling as if it were about to burst. This pain is always associated with, and presumably due to, a definite exacerbation of the hyperpiesis. In contradistinction to the true ache, it responds promptly to measures which lower the blood pressure. On the other hand, one is struck by the patients presenting 
systolic pressures of $200 \mathrm{~mm}$. of mercury or more who to the day of their death never complain of headache.

Palpitation, tachycardia, and inability to lie on the left side are again of great frequency. Of this triad palpitation needs the greatest emphasis, because it is a symptom found in assoriation with many functional nervous disorders, and the possibility of organic disease is apt to be overlooked.

Giddiness, which is usually dizziness, as opposed to true vertigo, may be a transient symptom produced by stosping or coughing, or it may be most persistent, and is then always associated with well-marked gencial arterio-sclerosis. Sudden attacks of definite vertigo are decidedly less common, and, if associated with vomiting, are to be regarded as of serious portent. Careful examination will usually reveal evidence of a central rervous lesion, prestimably a small haemorrhage, such as unilateral facial weakness and an extensor plantar response.

Noises in the ear are of two kinds. The one is in the nature of a low-pitched pulsation, and responds well to treatment; tho other, tinnitus, consists of various whistling, hissing, high-pitched sounds, and is present only if arterio-sclerosis is recognizable. Reduction of the hypelpiesis has no beneficial effect.

Anginal pain, though not uncommon, rarely gives rise to the intense paroxysms of anguish which characterize the discase, as generally recognized. Nevertheless I believe that we should accept the symptoms of substernal tension, or pressure, brought on by hurry, and relieved by rest, as definitely indicative of the presence of those factors necessary to produce erentually the fully developed syndrome. By insisting on a reduction of mental and physical activity we can thus postpone for many years the onset of a fatal seizure.

Mental symptoms are of diverse character, and on the whole not common. In the first place, our patient may complain of nervousness only, this srmptom being most common in the sallow female type. She is harassed by trivial imnoyances, and by fears based on no good cause, by palpitation, sleeplessness, and so on. She is only too apt to be placed in the category of functional nervous disorder, yet recovers from her symptoms with abatement of hyperpiesis.

The robust hyperpietics in the first few years of the clisease exhibit unusual mental vigour; but with the onset of arterio-sclerosis, drowsiness in the middle of the day may develop into a genuine cause for complaint. Failing memory, particularly for recent events, is a feature common to all classes, and advances in relation to structural changes of the cerebral vessels.

The most startling, but rare, change in mentality which I have met with has been a sudden onset of acute mania, the condition being invariably associated with a fall of blood pressure of considerable degrec-for example, from 220 to $130 \mathrm{~mm}$. of mercury. The psychosis continues until the hyperpiesis re-establishes itself. In the cases I have observel there has been no demonstrable physical change to be held accountable for the lowering of the tension.

Hacmorrhages, retinal and nasal, are of common occurrence and give rise to great anxiety. The former frequently leave permanent impairment of vision, but improvement in sight may take place over six months. Epistaxis is particularly alarming, owing to its profuseness and difficulty of control. Apart from this, it may be regarded as a blessing, possibly arerting a cerebral catastrophe. I have seen instances of melaena, haematemesis, and uterine haemorrhage for which no other cause than the existent hyperpiesis could be discovered. The possibility of this as the cause of sudden haemorrhage should, therefore, be borke in mind.

Pains in the limbs, and pain or a clutching sensation in the throat, are referable to the stretching of the local arteries by the excessive pressure of their contents. The latter symptom is particularly liable to be mistaken for one of functional origin.

1 hare not been impressed by symptoms referable to the urinary tract. In the later stages of the disease, when arterio-sclerosis is a pronounced feature, frequency of micturition is common. Apart from these circumstances, however, it is a rare symptom.

\section{Srans.}

Physical examination may reveal little more than a raised systolic pressure with a varying degree of exaltation of the diastolic reading. Before hypertrophy of the left ventricle is recognizable the most constant sign is the accentuation of the sound produced by the closure of the aortic cusps, together with a raising of its pitch. The last feature represents, I believe, atheromatous changes in the vessel with thickening of the valve segments. When absent the probability of "fictitions hyperpiesia" should be borne in mind.

The peripheral vessels show changes after variable periods, sooner in the thin and wiry individual than in the plethoric sufferer. When the radial artery appears to be normal to the examining fingers, the brachial is often grossly abnormal. The sensation imparted is that of a leathery toughness, rather than the hard pipe-stem eondition common in senility. The retinae should be examined in every case, for here may be found arterial disease long before it is apparent elsewhere.

The most constant change which $\mathrm{I}$ have found in the blood is that of a raised cholesterol content, ${ }^{2}$ often above $200 \mathrm{mg}$. per $100 \mathrm{c.cm}$. Whether this is of any etiological significance remains to be seen. In the meantime, it is of interest to note that there is an appreciahle increase of the substance in atheromatous vessels $;^{1}$ furthermore, I have notes of eighteen of this series suffering also from gallstones, a number too high to be explained on mere coincidence. My impression is that the highest cholesterol figures are obtainable from those hyperpietics exhibiting rapidly developing arterio-sclerosis. In a young woman exhibiting this feature to a remarkable degree a cholesteatoma was also remored from the skull. The erythrocyte count and blood urea are normal for many years. In the later stages a mild secondary anaemia and slight rise of urea may occur.

The urine in the majority of my cases was absolutely normal on repeated examinations. The appearance of a trace of albumin and a few hyaline casts is, of course, to be expected with advance of years and arterial disease.

The alimentary tract, as has been noted, gives rise to complaint in the lean individual on account of obstinate constipation. The tongue is commonly furred and visceroptosis is present. On the other hand, the plethoric individual has a clean tongue, powerful peristalsis, and high acid content of the gastric juice.

\section{Causes of Hypfrpiesia.}

In considering the possible causes of hyperpiesia we must accept a disturbance of the endocrine systen as the probable basis of our menopausal type. The association of menopause, obesity, loss of hair, sluggish mental processes, and rising blood pressure is too obtrusive to be overlooked, and this trpe of case responds well to treatment with thyroid extract. That overactivity of the suprarenal medulla may be a factor in the production of hyperpiesis has bern postulated, and it is of interest to note the increase of blood cholesterol which has been found in association with hyperpicsia and a hypertrophied adrenal cortex.

On the other hand, the young, lean, constipated patient with furred tongue and foul breath lends support to the theory that protein decomposition products are absorbed from the intestine and, failing to be destroyed in the liver, excrt a pressor influence on the systemic arterioles. True there is no conclusive evidence in support of this, but some of us may lean towards this explanation in view of the suggestive rlinical type.

The robust patient presents peculiar difficulties. Often he has bcen exceptionaliy athletic in youth, but has later assumed a solentary existence without at the same time reducing his intake of food. May it not be, therefore, that his powers of elimination are overtaxed, and that the basic fault is the same as in the previous group?

We must frankly admit that all these theories may be utterly wrong, yet as working hypotheses, and on the results of treatment, I feel they deserve more consideration than the view favouring the presence of a circulating pressor substance derived from diseased kidneys. To me 
it is inconceivable that renal disease could produce hyperpiesis of many. years' standing in the presence of a constantly normal urine.

Treatment

Exercise should bo restricted only in so far as is dictated by the patient's symptoms and signs. I mean by this that it is quite useless to send a patient to bed simply because he has a high blood pressure. Immobility may lower it 10 to $20 \mathrm{~mm}$. of mercury, seldom more, frequently less or not at all. Avoidance of hurry, especially after meals, of excitement, excessive exertion, and exposure to cold is naturally essential, particularly in the presence of anginal symptoms. These restrictions, however, interfere but little with the essential side of the patient's life, and are therefore all the more likely to be observed.

Diet.-Here, again, I believe that the prevalent custom of excluding all meat from the dietary is pointless. If we accept the theory of intestinal toxaemia, it may perliaps be reasonable to reduce the protein intake, but a restriction to meat thrice weekly is ample. On the other hand, eggs, brain, liver, and fat should be largely excluded in an attempt to lower the cholesterol in the circulation. In the obese glutton the diet should be a rigid one, and composed of fresh fruit, fish, and regetables. Alcohol, with the exception of light wines, should be forbidden.

Purgatives.-The daily use of saline cathartics is obnoxious and useless, possibly harmful in fact, by increasing the blood viscosity. Cases of obstinate constipation respond to a vegetarian diet in conjunction with abdominal massage and the exhibition of a non-irritating laxative such as sulphur, psyllium seeds, paraffin, or bemax.

Drugs.-We can dispose of the majority of those in common use with rapidity. Nitrites are valueless except in emergencies-in angina, for example-on account of the transitory nature of their action. Iodides I have never found of help in cases unassociated with syphilis. At one time it was believed that they lowered the viscosity of the blood and thus aided its flow. In order-to attain this effect the dosage must be enormous, while the digestive disturbances they produce are most objectionable. Bromides are of value in allaying restlessness and producing sleep in the nervous type of patient. Indirectly the blood pressure may thus be lowered. These salts are also of use in overcoming tinnitus. Thyroid extract is seldom helpful except in the obese menopausal patient. Here it can be highly efficacious, and should be prescribed in doses rising from $1 / 2$ grain thrice daily up to ten times this amount, provided that symptoms of intoxication do not arise.

Veratrum viride. We now come to the consideration of a drug with definite effect in reduction of hyperpiesis, the actual preparation used having been veratrone. A few years ago I employed this substance on a series of cases $^{2}$ and found that it reduced the blood pressure when administered by mouth or intravenously. In the former case the effect was variable even on the same patient, and it should be remembered that individnal susceptibilities also present great differences. Briefly the effect of intramuscular injection of $1 \mathrm{c.cm}$. is to lower the blood pressure from, say, $185 / 110$ to $105 / 63$ in half an hour, and the original level is regained in the course of one to two hours after administration. Such a big drop should, of course, be avoided, as it is attended by nausea, retching, and even collapse. On the other hand, $1 / 2 \mathrm{c.cm}$. resulted in a fall from $190 / 123$ to $120 / 70$ in half an hour in one patient, and from $200 / 130$ to $150 / 105$ in another. Given by the mouth $1 / 2$ to $1 \mathrm{c.cm}$. will in many cases lower the systolic pressure from 20 to $30 \mathrm{~mm}$. of mercury and keep it about this level for two hours. The drug may be regarded as of undoubted value, but it must be used with great caution in view of the possibility of idiosyncrasy. In very susceptible patients it may produce an alarming fall of blood pressure even if administered per os.

Acetylcholine, an acetyl ester of choline, stimulates the parasympathetic system and antagonizes adrenaline. Its effect in lowering arterial tension is produced by dilatation of the arterioles, the capillaries being unaffected. The actual preparation which I have used is " acécoline,"

which consists of acetylcholine hydrochloride stabilized with glucose. The resulting powder must be dissolved immediately before use, and the solution injected intramuscularly. Oral administration is useless, intravenous injection is extremely dangerous. As a precautionary measure, therefore, the piston of the syringe should be withdrawn before injection to ensure against an accidental delivery of the drug into the blood stream. The initial dose should be 0.05 gram, raised subsequently to 0.1 gram daily. The effect of the former dose is to produce a steady fall of systolic pressure, commencing within half an hour of administration, reaching its lowest level in three to four hours, and passing off gradually with a return to the former height in seven to nine hours after the injection; 0.1 gram produces its greatest effect in six to eight hours, and the previous pressure is often not reached until fifteen hours after administration. As examples may be cited the following results on the same patient, a female of 59 years, with hyperpiesia, normal urine, and moderate arteriosclerosis.

Time.

11.0 a.m

Acécoline $0 . .65$ gram injected.

12.30 p.m.

2.30 ,

$3.30 "$

$8.40 "$

$10.0 "$,

10.30 a.m

11.30 a.m

Acécoline 0.1 gram injected.

$4.15 \cdot \mathrm{p} . \mathrm{m}$

5.15 ,

6.15,

7.30,

8.30

9.15 ,

Although I have as yet used the drug in only a small number of cases, ret $I$ feel justified in drawing your attention to its action in the hope of gaining your co-operation in further investigations. It is clear that acetylcholine has a much more prolonged action than that of the drugs previously discussed, and it has the advantage of not producing suaden falls of pressure, which have caused the objectionable symptoms following the use of veratrone. On the other hand, the fact that oral administration is valueless is a decided point against its general adoption. We have yet to find whether a course of injections will produce a lasting effect on the hyperpiesis. There are already indications that it will do so, and some authors arlvise doses of 0.1 or even 0.2 gram night and morning for fiftcen consecutive days each month for thrce months.

You may object that by reducing the blood pressure we are merely dealing with a feature of the disease, rather than the cause. This is perfectly true; nevertheless it is justified in view of the fact that by so doing the leart and blood vessels are relieved of excessive stresses, at any rate for several hours in the day. The summation of such respites, taken over a year, must represent an enormous saving of effort to the heart muscle.

In advanced cases of hyperpiesia with extensive vascular discase a lowering of blood pressure of any extent is, of course, contraindicated, for it might result in depriving the narrowed cerebral vessels of their necessary blood supply. Judgement must therefore be used in choosing snitable subjects for this treatment.

Diathermy is of value in the treatment of hyperpiesia; in some a course produces a substantial reduction of blood pressure, and the improvement may persist for three or four months. On the whole, the results are disappointing.

Venesection is an old remedy, still unsurpassed in value by more modern measures. It is particularly appropriate for the obese, florid type of man who is unable, or unwilling, to carry out any dietary restrictions or consecutive treatment. The sallow patient often responds, however, equally well to frec bleeding. Headache, giddiness, and roaring in the ears vanish as if by magic, and the improvement is maintained for two or three months. The immo- 
diate result of venesection on the blood pressure is of ten very slight, and quite out of proportion to the alleviation of suffering; but during the following two or three days progressive fall may be observed. After this it may remain steady at its new level, or may rise again to its former height, yet the urgent symptoms do not return for many weeks.

Eliminative Treatment.

This is essentially a line of attack on the sallow, toxic type. For twelve consecutive days the patient is given 1/10 grain of pilocarpine hypodermically, followed by a hot wet pack, which is again followed by massage. Twice weekly during the course colon lavage is carried out. The diet during this time should consist of fresh fruit, vegetables, fish, brown bread, and butter. Before meals two tumblerfuls of Vichy water are to be taken. The beneficial effect on the patient's appearance, symptoms, and signs is very evident. The course may be repeated every two months, and can be carried out, if necessary, at home. Annual treatment at Harrogate is peculiarly suitable for this class of patient.

You will observe, then, that no hard-and-fast rule can be set for the treatment of hyperpiesia, but cach case must be considered in relation to type, symptoms, and physical signs. Given such discrimination, we can undoubtedly bring great relief to our patients, and, I believe, prolong life for the majority.

\section{Prognosis.}

Finally, the difficult problem of prognosis. You will agree that one great lesson learned in general practice is, particularly in cases of hyperpiesia, to give a cheerful outlook. Seven years ago I saw a lady with a blood pressure of 220/150, retinal and small cerebral haemorrhage, dyspnoea, obesity, and oedema of the ankles. Both types of haemorrhage were repeated within a month. It was thought unlikely that she would live two years, yet she is still highly active, never suffers from headache, and presents a systolic pressure of $220 \mathrm{~mm}$. or over.

As a general rule, a high diastolic pressure renders the prognosis more gloomy, as do also clear signs of cardiac or arterial disease. The menopausal type presents the most hopeful form, the lean patient the least favourable, on account of the earlier onset and rapid arterial degeneration. It is true, also, that the occurrence of retinal or cerebral lesions more of ten than not heralds the approach of a fatal termination.

Patients over 60 years of age survive in the most astounding manner in spite of the presence of some, or all, of the signs usually indicating a bad prognosis. The outstanding example in my practice was that of a man of 70 , known to have had a high blood pressure for at least thirty years. On three occasions he developed auricular fibrillation, which was overcome by quinidine, and once he developed diarrhoea, incontinence of urine, pyrexia, petechial haemorrhages, and a streptococcus was recovered from his blood. And yet he lives!

The anginal pains to which $I$ have referred are not of the same gloomy significance when a high blood pressure is present as when it is absent. They disappear with reduction of hyperpiesis.

In all cases we should, of course, warn the relatives of the possibility of cardiac failure, coronary thrombosis, fatal angina, and cerebral haemorrhage. The patient, on the other hand, should be encouraged, as far as is consistent with ensuring his attention to our therapeutic efforts.

1 Allbutt, T. C.: Arteriosclerosis: A Summary View, 1925.

$s$ Villaret, M., and Justin-Besancon, L.: Lancet, 1929, i, p. 493.

\section{BLINDNESS IN OLD AGE.* BY}

J. JAMESON EVANS, M.D., C.M., F.R.C.S., HONORARY OPHTHALMIC SURGEON, ROYAL INSTITUTE FOR THE BLIND, BIRMINGHAM; HONORARY SURGEON, BIRMINGHAM AND MIDLAND EYE HOSPITAL.

Under the provisions of the Blind Persons Act of 1920 it was enacted that " Every blind person who has attained the age of fifty shall be entitled to receive and to continue to receive such pension as, under the Old Age Pensions Acts, 1908 to 1919, he would be entitled to receive if he had attained the age of seventy," and the provisions of those Acts, including the provisions as to expenses, shall apply in all respects to such person as if for the first statutory condition there were substituted a condition that the person must have attained the age of fifty, and be so blind as to be unable to perform any work for which eyesight is essential, and as if for reference to "seventy" and "fifty" there were respectively substituted references to "fifty" and " thirty."

The Act further states that "it shall be the duty of every county and every county borough ... to make arrangements to the satisfaction of the Minister of Health for promoting the welfare of blind persons ordinarily resident within their area, and such council may for this purpose provide and maintain or contribute towards the provision and maintenance of workshops, hostels, houses o! other places for the reception of blind persons. . . The expenses incurred by a council under this section shall be defrayed ... out of the county or borough fund or rate."

It will be observed that the Act contains no specific arrangement for the certification of blind persons. Possibly those who drafted the Act considered that an applicant for a blind pension was in as good a position to provide a certificate of blindness as the old age pensioner was in providing a birth certificate. Possibly the "approved

* Read in the Section of Ophthalmology at the Annual Meeting of the British Medical Association, Manchester, 1929. voluntary agencies" which administer the funds provided for the benefit of the blind might have claimed a grant for the purpose of certification from the Ministry of Health on the grounds of " necessity for securing the registration of all blind persons" (Rule 32, Ministry of Health Regulations and Rules for the Welfare of the Blind, August 7th, 1919).

As far as I know, however, this has not been done, and the local authorities have found it necessary, in the interest of the ratepayer, to get applicants for blind pensions or grants examined and authoritatively certified before the pension or grant is allowed. The Ministry of Health is very exacting with regard to the certification of trainees, and demands a medical certificate giving the visual acuity in each eye, the nature and extent of the visual defects present, and a definite opinion, formed after due consideration of all the visual conditions, that the person is too blind to perform work for which eyesight is essential. The local authority in Birmingham only requires a statement that the applicant is or is not blind within the meaning of the Blind Persons Act. That there is need for caution and discrimination in the administration of this Act is shown by the fact that a sum of $£ 10,470$ was distributed in weekly and special grants by the voluntary agency in Birmingham last year. The number of pensioners is rapidly increasing. In Birmingham in 1919 the number of adult blind living in their own homes was 425 , whereas this year they number 1,005. When it is remembered that since 1928 every blind person may have his income made up to $£ 1$ a week, it is obvious that the expense to the community will be very considerable, and I think that the certification of blind pensioners should be no less detailed and comprehensive than that demanded by the Ministry of Health in the case of trainees. Since 1928 the number of applicants has more than doubled as compared with the years $1924-27$.

The purpose of this paper, however, is not in the main to criticize the Blind Persons Act and its administration, but to analyse the causes of blindness in 700 old people as certified by me for the local voluntary agency in Birmingham during the last four and a half years. I have 